\title{
Knowledge and Attitude towards Sexually \\ Transmitted Disease and HIV/AIDS among Secondary \\ Level School Students
}

Urmila Sunuwar ${ }^{1}$

\begin{abstract}
Adolescent sexuality leads to adolescent pregnancy, unsafe abortion, reproductive tract infections, sexually transmitted infections and HIV/AIDS. The study objective is to assess the knowledge and attitude towards sexually transmitted disease and HIV/AIDs among secondary level school students of Kathmandu metropolitan city. Descriptive cross-sectional study was conducted by using self-administered questionnaires. The study population was school adolescent students. They were selected by using random sampling method. After data collection, descriptive statistics were used to analyse the collected data. Knowledge and attitude towards sexual transmitted disease is significantly varied with student's background characteristics. Around 95\% of study population had heard about sexually transmitted disease and almost 99\% heard about HIV/AIDS. Majority 85.6\% of students reported that they "agreed" that HIV/AIDS is a major problem in Nepal however $14.4 \%$ students were not agreed on it. $73 \%$ respondents reported that teachers are the main source of knowledge regarding sexual transmitted disease and HIV/AIDS.It is clear that students need a specific package of education about sexual and reproductive health in school curriculum so that they could gain more information about sexually transmitted disease and HIV/AIDS.
\end{abstract}

Keywords: Knowledge, attitude, sexually transmitted, diseases, adolescent

\section{Introduction}

School students are often called as the generation of hope. It plays an important role for the present as well as future health status of a country (Jain et al., 2016). Spreading knowledge and awareness towards the adolescent students about sexually transmitted diseases and HIV is one of the key strategies utilized in the prevention and control of HIV/AIDS worldwide.

1 Ms. Sunuwar is a lecturer of Population Education at Koteshwor Multiple Campus, Koteshwor, Kathmandu affiliated to T. U. 
Inadequate knowledge and risky practices are major hindrances in preventing the spread of HIV. In many countries, sexually transmitted diseases (STD) and unplanned pregnancies are frequently observed among adolescents (Alhasawi et al., 2019). Sexual transmitted diseases (STDs), refers to the disorder that are generally spread by unsafe sexual contact, (DeCherney et al., 2007). Most of them are initially asymptomatic, and increases the risk of transmission to others (Murry, Rosenthal and Pfaller, 2013). It is considered as a major public health problem worldwide, especially in the developing countrieslikeNepal. Similarly, HIV/AIDS is a global epidemic and is considered one of the greatest public health problems both in developed and developing world (Poudel et al., 2015). Most of the adolescent in Nepalhave little knowledgeaboutsexual transmitted diseases including HIV/AIDS. So, adolescent's sexual health is poor due to the lack of proper information on sexual transmitted disease and HIV/AIDS. Adolescents at schools are more likely to have experienced many traumatic events, including neglected sexually, physically and emotionally abused. This may distort their understanding on sex, sexuality and personal relationship which result in low self-esteem and inappropriate sexual behavior. They may lack the necessary skills and confidence to negotiate and sustain positive personal relationships. Young people need consistent guidance and support about sex and relationship from their parents but the parents are less likely to do so because of our socio-cultural thoughts. Sex is one of the commonest human behaviors. However, in Nepalese society people don't enjoy talking about it, they talk very little about the sex or sex education. It is a subject that is considered to be a very confidential matter because of social taboos and wrong impression about sex and sexual organs. Young and adults are not only ignorant about sex, sexuality and reproductive health, but also have a misconception on it. Thus, this study is to assess the knowledge and attitude towards sexual transmitted disease and HIV/ AIDs among secondary level school students of Kathmandu metropolitan city.

\section{Methodology}

The present study was descriptive and cross sectional in nature. This study was carried out in six secondary level schools of Kathmandu Metropolitan City. It covered grade 9 students of selected schools. Thirty students (15 boys and 15 girls) were chosen from each school. So, the total sample size was 180 adolescent students ( 90 boys and 90 girls). 90 students (45 boys and 45 girls) were from the public school and remaining 90 (45 boys and 45 girls) students were from the private school. Random sampling technique was used to select the school and students. List of the all students who were 
studying in grade 9 were prepared separately for male and female in the day of data collection. From the list, 15 male and 15 female students were selected randomly. A set of self-administered questionnaire was developed and used. Boys and girls students were kept in separate class. Attention was paid against the possibility of coping from their peers. Researcher conducted and monitored all these tasks. A set of questionnaire was distributed to the students and requested to administer the questionnaire. After data collection, descriptive statistics were used to analyze the collected data.

\section{Results and Discussion}

\section{Background Characteristics of Students}

A total of 180 students of grade 9 were included in this study. Among them 90 students were from Private school and the rest (90 students) were from public school. As presented in table below, regarding the age group, around $(26.7 \%)$ students $(22.2 \%$ male and $31.1 \%$ female) of private school were age of below 15 years, $60.6 \%$ students (65.6\% male and $55.6 \%$ female) were age of $15-16$ years, and 17 and above were $12.8 \%$ (12.2\% male and $13.3 \%$ female). Similarly, it was found that students from public school were older than students from private school. For instance, more than fifty (23\%) students were aged 17 and above were from public school while the percentage of students aged 17 and above and studying in private school was very low (2.2\%).

Table 1: Background characteristics of students

\begin{tabular}{|l|l|r|r|r|r|r|r|}
\hline \multicolumn{2}{|c|}{} & \multicolumn{2}{c|}{ Male } & \multicolumn{2}{c|}{ Female } & \multicolumn{2}{c|}{ Total } \\
\cline { 3 - 8 } \multicolumn{2}{|c|}{ Type of school } & \multicolumn{1}{c|}{$\%$} & \multicolumn{1}{c|}{$\mathrm{N}$} & \multicolumn{1}{c|}{$\%$} & \multicolumn{1}{c|}{$\mathrm{N}$} & \multicolumn{1}{c|}{$\%$} & \\
\hline \multirow{5}{*}{ Caste } & Private school & 45 & 25.0 & 45 & 25.0 & 90 & 50.0 \\
\cline { 2 - 8 } & Public school & 45 & 25.0 & 45 & 25.0 & 90 & 50.0 \\
\hline & Newar & 15 & 8.3 & 10 & 5.6 & 25 & 13.9 \\
\cline { 2 - 8 } & Chhetri & 32 & 17.8 & 18 & 10.0 & 50 & 27.8 \\
\cline { 2 - 8 } & Brahman & 14 & 7.8 & 29 & 16.1 & 43 & 23.9 \\
\cline { 2 - 8 } & Janajati & 22 & 12.2 & 22 & 12.2 & 44 & 24.4 \\
\cline { 2 - 8 } & Dalit & 1 & .6 & 7 & 3.9 & 8 & 4.4 \\
\cline { 2 - 8 } & Madheshi & 6 & 3.3 & 4 & 2.2 & 10 & 5.6 \\
\hline
\end{tabular}




\begin{tabular}{|c|c|c|c|c|c|c|c|}
\hline \multirow{6}{*}{ Age } & 13 & 4 & 2.2 & 3 & 1.7 & 7 & 3.9 \\
\hline & 14 & 16 & 8.9 & 25 & 13.9 & 41 & 22.8 \\
\hline & 15 & 29 & 16.1 & 30 & 16.7 & 59 & 32.8 \\
\hline & 16 & 30 & 16.7 & 20 & 11.1 & 50 & 27.8 \\
\hline & 17 & 7 & 3.9 & 6 & 3.3 & 13 & 7.2 \\
\hline & 18 & 4 & 2.2 & 6 & 3.3 & 10 & 5.6 \\
\hline \multirow{4}{*}{ Religion } & Hindu & 75 & 41.7 & 73 & 40.6 & 148 & 82.2 \\
\hline & Buddha & 12 & 6.7 & 13 & 7.2 & 25 & 13.9 \\
\hline & Kristain & 1 & .6 & 2 & 1.1 & 3 & 1.7 \\
\hline & Kirat & 2 & 1.1 & 2 & 1.1 & 4 & 2.2 \\
\hline Marital status & Single & 90 & 50.0 & 90 & 50.0 & 180 & 100.0 \\
\hline \multirow{2}{*}{ type of family } & Nuclear & 63 & 35.0 & 72 & 40.0 & 135 & 75.0 \\
\hline & Extended & 27 & 15.0 & 18 & 10.0 & 45 & 25.0 \\
\hline \multirow{4}{*}{ Living with } & Family & 68 & 37.8 & 71 & 39.4 & 139 & 77.2 \\
\hline & Friend & 4 & 2.2 & 1 & .6 & 5 & 2.8 \\
\hline & Relatives & 16 & 8.9 & 18 & 10.0 & 34 & 18.9 \\
\hline & Alone & 2 & 1.1 & & & 2 & 1.1 \\
\hline \multirow{4}{*}{$\begin{array}{l}\text { Source of income } \\
\text { for the family }\end{array}$} & Agriculture & 19 & 10.6 & 17 & 9.4 & 36 & 20.0 \\
\hline & Business & 39 & 21.7 & 21 & 11.7 & 60 & 33.3 \\
\hline & Job & 28 & 15.6 & 35 & 19.4 & 63 & 35.0 \\
\hline & Other & 4 & 2.2 & 17 & 9.4 & 21 & 11.7 \\
\hline \multirow{4}{*}{ monthly income } & $5000-8000$ & 19 & 10.6 & 24 & 13.3 & 43 & 23.9 \\
\hline & $9000-12000$ & 19 & 10.6 & 8 & 4.4 & 27 & 15.0 \\
\hline & $13000-16000$ & 20 & 11.1 & 13 & 7.2 & 33 & 18.3 \\
\hline & above 16000 & 32 & 17.8 & 45 & 25.0 & 77 & 42.8 \\
\hline \multirow{2}{*}{$\begin{array}{l}\text { Father literacy } \\
\text { status }\end{array}$} & Yes & 75 & 41.7 & 71 & 39.4 & 146 & 81.1 \\
\hline & No & 15 & 8.3 & 19 & 10.6 & 34 & 18.9 \\
\hline \multirow{2}{*}{$\begin{array}{l}\text { Mother literacy } \\
\text { status }\end{array}$} & Yes & 54 & 30.0 & 58 & 32.2 & 112 & 62.2 \\
\hline & No & 36 & 20.0 & 32 & 17.8 & 68 & 37.8 \\
\hline
\end{tabular}




\begin{tabular}{|l|l|r|r|r|r|r|r|}
\hline \multirow{4}{*}{ Radio Exposure } & Frequently & 18 & 10.0 & 19 & 10.6 & 37 & 20.6 \\
\cline { 2 - 8 } & Some Time & 58 & 32.2 & 51 & 28.3 & 109 & 60.6 \\
\cline { 2 - 8 } & Very Less & 10 & 5.6 & 12 & 6.7 & 22 & 12.2 \\
\cline { 2 - 8 } & Never & 4 & 2.2 & 8 & 4.4 & 12 & 6.7 \\
\hline \multirow{4}{*}{ TV Exposure } & Frequently & 53 & 29.4 & 63 & 35.0 & 116 & 64.4 \\
\cline { 2 - 8 } & Some Time & 32 & 17.8 & 21 & 11.7 & 53 & 29.4 \\
\cline { 2 - 8 } & Very Less & 5 & 2.8 & 6 & 3.3 & 11 & 6.1 \\
\hline \multirow{3}{*}{$\begin{array}{l}\text { Newspaper Expo- } \\
\text { sure }\end{array}$} & Frequently & 40 & 22.2 & 33 & 18.3 & 73 & 40.6 \\
\cline { 2 - 8 } & Some Time & 43 & 23.9 & 45 & 25.0 & 88 & 48.9 \\
\cline { 2 - 8 } & Very Less & 5 & 2.8 & 11 & 6.1 & 16 & 8.9 \\
\cline { 2 - 8 } & Never & 2 & 1.1 & 1 & .6 & 3 & 1.7 \\
\hline \multirow{2}{*}{ Total } & 90 & 50.0 & 90 & 50.0 & 180 & 100.0 \\
\hline
\end{tabular}

Overall more than a fourth students were from Chhetri (28\%) followed by Janajati (24.4\%) and Brahmin (23.9\%). Similarly, 13.9\% students (16.7\% male and $11.1 \%$ female) were from Newar caste; $5.6 \%$ (6.7\% male and $4.4 \%$ female) were Madheshi and 4.4\% (1.1\% male and 7.8\% female) were Dalit. In regards to caste, not much difference was observed between private and public school students.

It was found that overall more than four in five students of private schools $(82.2 \%)$ were reported that they follow Hindu religion, 13.9\% follow Buddha religion, 2.2\% follow Kirat religion and $1.7 \%$ follow Christianity.Overall three of fourth $(75.0 \%)$ of the students (70.0\% male and $80.0 \%$ female) lived in nuclear family and the rest of the students (24.4\%) live in extended family.In the family size of students, about two-fifths (36.7\%) students $(28.9 \%$ male and $44.4 \%$ female) have the family size below 4 members while about a tenth students $(8.9 \%)$ had more than 8 family members. Overall almost all student respondents $(96.1 \%)$ have the access of any type of communication medium. This was reported $95.6 \%$ male students and $100 \%$ female students who studying in private school had the access of communication means and $95.6 \%$ male students and $93.3 \%$ female students who are studying in public school had such access. Similarly, overall $20.0 \%$ students $(20.0 \%$ male and $21.2 \%$ female) reported that they listened to radio frequently while $60.6 \%$ students ( $64.4 \%$ male and $56.7 \%$ female) replied sometime. It is notable that almost two-thirds student watched TV frequently. A higher percentage of students who are studying in private school (76\%) than public school $(62 \%)$ reported that they watched 
TV frequently. On the other hand, a higher percentage of students who were studying in public school $(10 \%)$ than private school $(2 \%)$ reported that they watched TV rarely Newspaper exposure is low compared with TV and radio exposure.

\section{Knowledge about Sexually Transmitted Disease}

Students were asked whether they heard about sexually transmitted disease or not. It was found that overwhelming majority of them (95\%) heard of sexually transmitted disease. A slightly higher percentage of male (96\%) than female (94\%) reported that they heard sexually transmitted disease. In the current study, knowledge about STD was grouped into three subtype i.e. Transmitted by facing the person, Transmitted by sexual intercourse and both of them. It is encouraging to note that almost all students (95\%) reported that STD is transmitted by sexual intercourse. A higher percentage of students in public school than private school reported it. Very few other students reported that it can be transmitted by talking with person and both of the issue (by talking and by sexual intercourse).

Table 2: Percentage distribution of students according to their knowledge about Sexual transmitted diseases

\begin{tabular}{|l|r|r|r|r|r|r|r|r|r|}
\hline & \multicolumn{2}{|c|}{ Private school } & \multicolumn{2}{c|}{ Public school } & \multicolumn{3}{c|}{ All Total } \\
\cline { 2 - 10 } & Male & Female & Total & Male & Female & Total & Male & Female & Total \\
\hline $\begin{array}{l}\text { Heard about Sexually } \\
\text { Transmitted Diseases }\end{array}$ & & & & & & & & & \\
\hline Yes & 97.8 & 91.1 & 94.4 & 93.3 & 97.8 & 95.6 & 95.6 & 94.4 & 95.0 \\
\hline No & 2.2 & 8.9 & 5.6 & 6.7 & 2.2 & 4.4 & 4.4 & 5.6 & 5.0 \\
\hline Total & $\mathbf{1 0 0}$ & $\mathbf{1 0 0}$ & $\mathbf{1 0 0}$ & $\mathbf{1 0 0}$ & $\mathbf{1 0 0}$ & $\mathbf{1 0 0}$ & $\mathbf{1 0 0}$ & $\mathbf{1 0 0}$ & $\mathbf{1 0 0}$ \\
\hline N & $\mathbf{4 5}$ & $\mathbf{4 5}$ & $\mathbf{9 0}$ & $\mathbf{4 5}$ & $\mathbf{4 5}$ & $\mathbf{9 0}$ & $\mathbf{9 0}$ & $\mathbf{9 0}$ & $\mathbf{1 8 0}$ \\
\hline STD is.. & & & & & & & & & \\
\hline $\begin{array}{l}\text { Transmitted by talking } \\
\text { with person }\end{array}$ & 4.5 & & 2.4 & & & & 2.3 & & 1.2 \\
\hline $\begin{array}{l}\text { Transmitted by sexual } \\
\text { intercourse }\end{array}$ & 86.4 & 97.6 & 91.8 & 97.6 & 97.7 & 97.7 & 91.9 & 97.6 & 94.7 \\
\hline All of the them & 9.1 & 2.4 & 5.9 & 2.4 & 2.3 & 2.3 & 5.8 & 2.4 & 4.1 \\
\hline Total & $\mathbf{1 0 0}$ & $\mathbf{1 0 0}$ & $\mathbf{1 0 0}$ & $\mathbf{1 0 0}$ & $\mathbf{1 0 0}$ & $\mathbf{1 0 0}$ & $\mathbf{1 0 0}$ & $\mathbf{1 0 0}$ & $\mathbf{1 0 0}$ \\
\hline N & $\mathbf{4 4}$ & $\mathbf{4 1}$ & $\mathbf{8 5}$ & $\mathbf{4 2}$ & $\mathbf{4 4}$ & $\mathbf{8 6}$ & $\mathbf{8 6}$ & $\mathbf{8 5}$ & $\mathbf{1 7 1}$ \\
\hline
\end{tabular}




\section{Knowledge about HIV/AIDS}

It is notable that all male and female in both private and public school except 2 percent male in public school reported that they heard about HIV/AIDS. Students reported sources of knowledge of HIV/AIDS such as through "Radio", "TV", "Newspaper", "friends", "teacher", "local health workers", "health post", and "other" sources. Teachers as source of HIV/AIDS information were reported by more than two thirds of students $(73 \%)$. A higher percentage of students in private school $(80 \%)$ than public school (66\%) reported the teacher as a source of HIV information. Almost a fifth student (18\%) reported the source of HIV information was TV. Similarly, few students reported radio $(6 \%)$, friends $(6 \%)$ newspaper $(4 \%)$ etc.

Table 3: Percentage distribution of students according to their awareness of HIV/AIDS and source of knowledge

\begin{tabular}{|l|r|r|r|r|r|r|r|r|r|}
\hline \multirow{2}{*}{} & \multicolumn{2}{|c|}{ Private school } & \multicolumn{2}{c|}{ Public school } & \multicolumn{3}{c|}{ All Total } \\
\cline { 2 - 11 } & Male & Female & \multicolumn{1}{|c|}{ Total } & Male & Female & Total & Male & Female & Total \\
\hline HIV/AIDS & & & & & & & & & \\
\hline Yes & 100 & 100 & 100 & 97.8 & 100 & 98.9 & 98.9 & 100 & 99.4 \\
\hline No & & & & 2.2 & & 1.1 & 1.1 & & .6 \\
\hline Total & $\mathbf{1 0 0}$ & $\mathbf{1 0 0}$ & $\mathbf{1 0 0}$ & $\mathbf{1 0 0}$ & $\mathbf{1 0 0}$ & $\mathbf{1 0 0}$ & $\mathbf{1 0 0}$ & $\mathbf{1 0 0}$ & $\mathbf{1 0 0}$ \\
\hline N & $\mathbf{4 5}$ & $\mathbf{4 5}$ & $\mathbf{9 0}$ & $\mathbf{4 5}$ & $\mathbf{4 5}$ & $\mathbf{9 0}$ & $\mathbf{9 0}$ & $\mathbf{9 0}$ & $\mathbf{1 8 0}$ \\
\hline $\begin{array}{l}\text { Source of knowl- } \\
\text { edge* }\end{array}$ & & & & & & & & & \\
\hline Radio & 2.2 & 4.4 & 3.3 & 8.9 & 8.9 & 8.9 & 5.6 & 6.7 & 6.1 \\
\hline TV & 31.1 & 2.2 & 16.7 & 15.6 & 24.4 & 20.0 & 23.3 & 13.3 & 18.3 \\
\hline Newspaper & & 2.2 & 1.1 & 8.9 & 6.7 & 7.8 & 4.4 & 4.4 & 4.4 \\
\hline Friends & 6.7 & 8.9 & 7.8 & 8.9 & & 4.4 & 7.8 & 4.4 & 6.1 \\
\hline Teachers & 77.8 & 82.2 & 80.0 & 60.0 & 71.1 & 65.6 & 68.9 & 76.7 & 72.8 \\
\hline $\begin{array}{l}\text { Local health } \\
\text { workers }\end{array}$ & & 2.2 & 1.1 & 4.4 & 4.4 & 4.4 & 2.2 & 3.3 & 2.8 \\
\hline Health post & & 2.2 & 1.1 & 6.7 & & 3.3 & 3.3 & 1.1 & 2.2 \\
\hline Other & 4.4 & 3.3 & & & & 1.1 & 2.2 & 1.7 \\
\hline N & & $\mathbf{4 5}$ & $\mathbf{9 0}$ & $\mathbf{4 5}$ & $\mathbf{4 5}$ & $\mathbf{9 0}$ & $\mathbf{9 0}$ & $\mathbf{9 0}$ & $\mathbf{1 8 0}$ \\
\hline
\end{tabular}


* Percentages total may exceed 100 due to multiple responses

\section{Knowledge on HIV Transmission}

Knowledge about HIV transmission was assessed by asking whether HIV can be transmitted through sexual intercourse using condom, sharing the food, and mosquito bite. It is notable that most of the students in both public and private school had correct knowledge on HIV transmission. For instance, majority of the students $(90 \%)$ reported that HIV cannot be transmitted by sexual intercourse if the person has used condom in every sexual intercourse. A higher percentage of both male and female students of private school had more knowledge about it than students of public school. Similarly, more than four-fifth (83\%) reported that HIV/AIDS can not be transmitted by sharing food with infected person. However, almost a tenth student had misconception on it. It is notable that misconception regarding mosquito bite and HIV transmission was high among students. For instance, more than three in five students (62\%) reported that HIV AIDS can be transmitted through mosquito bite. The percentage of female who have reported mosquito bite was higher than male students in both private and public school.

Table 4: Background characteristics of students by knowledge on HIV transmission

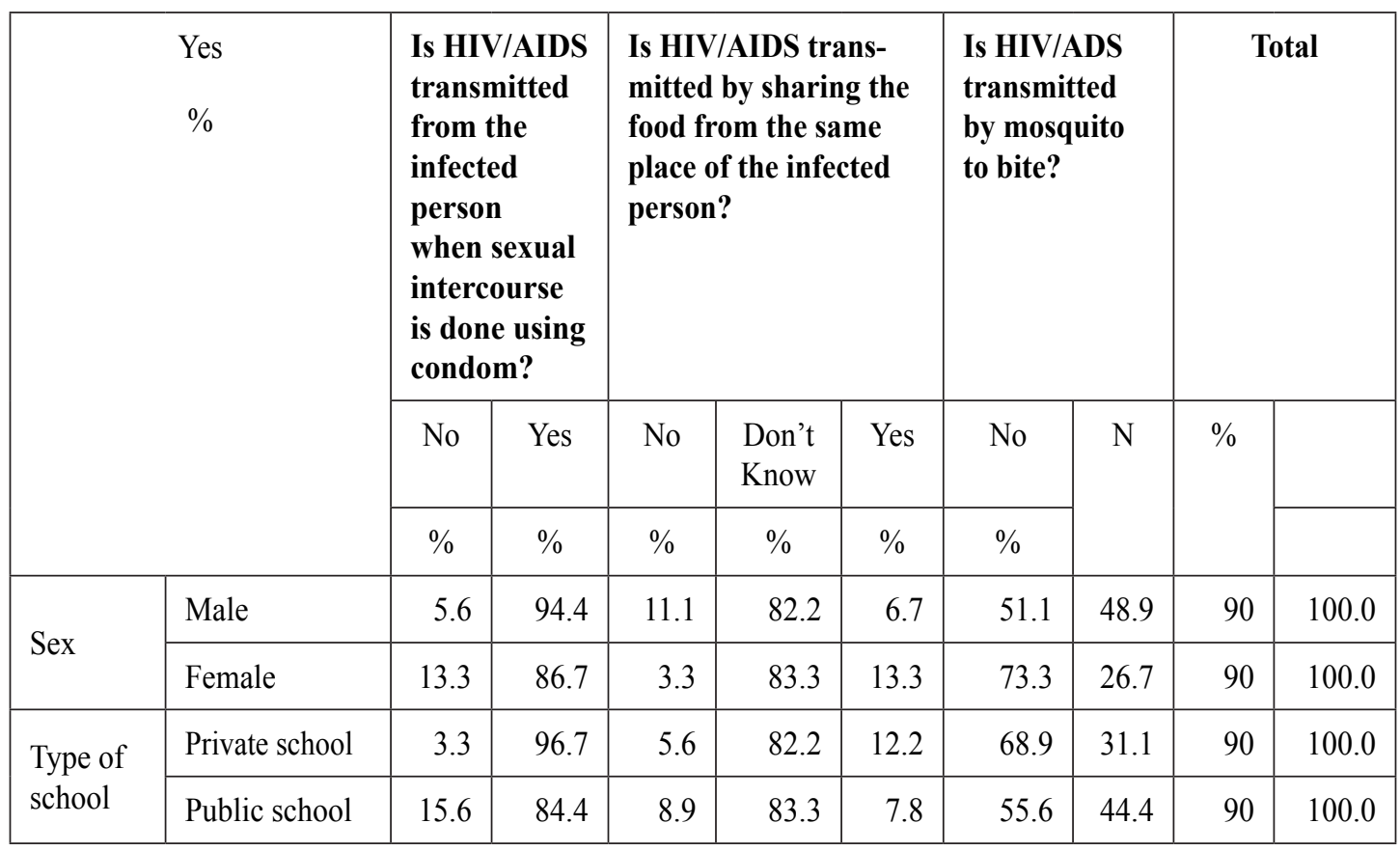




\begin{tabular}{|c|c|c|c|c|c|c|c|c|c|c|}
\hline \multirow{6}{*}{ Caste } & Newar & 4.0 & 96.0 & & 80.0 & 20.0 & 56.0 & 44.0 & 25 & 100.0 \\
\hline & Chhetri & 10.0 & 90.0 & 16.0 & 80.0 & 4.0 & 62.0 & 38.0 & 50 & 100.0 \\
\hline & Brahman & 11.6 & 88.4 & 4.7 & 83.7 & 11.6 & 60.5 & 39.5 & 43 & 100.0 \\
\hline & Janajati & 9.1 & 90.9 & 4.5 & 84.1 & 11.4 & 70.5 & 29.5 & 44 & 100.0 \\
\hline & Dalit & & 100.0 & & 100.0 & & 62.5 & 37.5 & 8 & 100.0 \\
\hline & Madheshi & 20.0 & 80.0 & 10.0 & 80.0 & 10.0 & 50.0 & 50.0 & 10 & 100.0 \\
\hline \multirow{6}{*}{ Age } & 13 & 14.3 & 85.7 & & 85.7 & 14.3 & 57.1 & 42.9 & 7 & 100.0 \\
\hline & 14 & 7.3 & 92.7 & 7.3 & 80.5 & 12.2 & 75.6 & 24.4 & 41 & 100.0 \\
\hline & 15 & 8.5 & 91.5 & 5.1 & 84.7 & 10.2 & 62.7 & 37.3 & 59 & 100.0 \\
\hline & 16 & 8.0 & 92.0 & 8.0 & 84.0 & 8.0 & 52.0 & 48.0 & 50 & 100.0 \\
\hline & 17 & 15.4 & 84.6 & 15.4 & 76.9 & 7.7 & 53.8 & 46.2 & 13 & 100.0 \\
\hline & 18 & 20.0 & 80.0 & 10.0 & 80.0 & 10.0 & 70.0 & 30.0 & 10 & 100.0 \\
\hline \multirow{4}{*}{ Religion } & Hindu & 9.5 & 90.5 & 7.4 & 83.1 & 9.5 & 60.8 & 39.2 & 148 & 100.0 \\
\hline & Buddha & 12.0 & 88.0 & 4.0 & 84.0 & 12.0 & 68.0 & 32.0 & 25 & 100.0 \\
\hline & Kristain & & 100.0 & & 66.7 & 33.3 & 33.3 & 66.7 & 3 & 100.0 \\
\hline & Kirat & & 100.0 & 25.0 & 75.0 & & 100.0 & & 4 & 100.0 \\
\hline $\begin{array}{l}\text { Marital } \\
\text { status }\end{array}$ & Single & 9.4 & 90.6 & 7.2 & 82.8 & 10.0 & 62.2 & 37.8 & 180 & 100.0 \\
\hline \multirow{2}{*}{$\begin{array}{l}\text { Type of } \\
\text { family }\end{array}$} & Nuclear & 10.4 & 89.6 & 5.9 & 82.2 & 11.9 & 63.0 & 37.0 & 135 & 100.0 \\
\hline & Extended & 6.7 & 93.3 & 11.1 & 84.4 & 4.4 & 60.0 & 40.0 & 45 & 100.0 \\
\hline \multirow{4}{*}{$\begin{array}{l}\text { Living } \\
\text { with }\end{array}$} & Family & 8.6 & 91.4 & 4.3 & 84.9 & 10.8 & 60.4 & 39.6 & 139 & 100.0 \\
\hline & Friend & & 100.0 & & 100.0 & & 40.0 & 60.0 & 5 & 100.0 \\
\hline & Relatives & 11.8 & 88.2 & 17.6 & 73.5 & 8.8 & 70.6 & 29.4 & 34 & 100.0 \\
\hline & Alone & 50.0 & 50.0 & 50.0 & 50.0 & & 100.0 & & 2 & 100.0 \\
\hline \multirow{4}{*}{$\begin{array}{l}\text { Source } \\
\text { of } \\
\text { income } \\
\text { for the } \\
\text { family }\end{array}$} & Agriculture & 13.9 & 86.1 & 19.4 & 69.4 & 11.1 & 61.1 & 38.9 & 36 & 100.0 \\
\hline & Business & 5.0 & 95.0 & 8.3 & 80.0 & 11.7 & 65.0 & 35.0 & 60 & 100.0 \\
\hline & Job & 7.9 & 92.1 & 1.6 & 87.3 & 11.1 & 55.6 & 44.4 & 63 & 100.0 \\
\hline & Other & 19.0 & 81.0 & & 100.0 & & 76.2 & 23.8 & 21 & 100.0 \\
\hline
\end{tabular}




\begin{tabular}{|c|c|c|c|c|c|c|c|c|c|c|}
\hline \multirow{4}{*}{$\begin{array}{l}\text { Monthly } \\
\text { income }\end{array}$} & $5000-8000$ & 11.6 & 88.4 & 16.3 & 74.4 & 9.3 & 65.1 & 34.9 & 43 & 100.0 \\
\hline & $9000-12000$ & 14.8 & 85.2 & & 92.6 & 7.4 & 40.7 & 59.3 & 27 & 100.0 \\
\hline & $13000-16000$ & 18.2 & 81.8 & 3.0 & 84.8 & 12.1 & 48.5 & 51.5 & 33 & 100.0 \\
\hline & above 16000 & 2.6 & 97.4 & 6.5 & 83.1 & 10.4 & 74.0 & 26.0 & 77 & 100.0 \\
\hline \multirow{2}{*}{$\begin{array}{l}\text { Father } \\
\text { literacy } \\
\text { status }\end{array}$} & Yes & 8.2 & 91.8 & 4.8 & 84.2 & 11.0 & 61.6 & 38.4 & 146 & 100.0 \\
\hline & No & 14.7 & 85.3 & 17.6 & 76.5 & 5.9 & 64.7 & 35.3 & 34 & 100.0 \\
\hline \multirow{2}{*}{$\begin{array}{l}\text { Mother } \\
\text { literacy } \\
\text { status }\end{array}$} & Yes & 5.4 & 94.6 & 4.5 & 83.9 & 11.6 & 59.8 & 40.2 & 112 & 100.0 \\
\hline & No & 16.2 & 83.8 & 11.8 & 80.9 & 7.4 & 66.2 & 33.8 & 68 & 100.0 \\
\hline \multirow{4}{*}{$\begin{array}{l}\text { Radio } \\
\text { exposure }\end{array}$} & Frequently & 10.8 & 89.2 & 8.1 & 78.4 & 13.5 & 48.6 & 51.4 & 37 & 100.0 \\
\hline & Some Time & 7.3 & 92.7 & 6.4 & 89.0 & 4.6 & 64.2 & 35.8 & 109 & 100.0 \\
\hline & Very Less & 13.6 & 86.4 & 13.6 & 81.8 & 4.5 & 68.2 & 31.8 & 22 & 100.0 \\
\hline & Never & 16.7 & 83.3 & & 41.7 & 58.3 & 75.0 & 25.0 & 12 & 100.0 \\
\hline \multirow{3}{*}{$\begin{array}{l}\text { TvEx- } \\
\text { pousre }\end{array}$} & Frequently & 7.8 & 92.2 & 2.6 & 86.2 & 11.2 & 59.5 & 40.5 & 116 & 100.0 \\
\hline & Some Time & 11.3 & 88.7 & 17.0 & 73.6 & 9.4 & 67.9 & 32.1 & 53 & 100.0 \\
\hline & Very Less & 18.2 & 81.8 & 9.1 & 90.9 & & 63.6 & 36.4 & 11 & 100.0 \\
\hline \multirow{4}{*}{$\begin{array}{l}\text { News- } \\
\text { paper } \\
\text { exposure }\end{array}$} & Frequently & 6.8 & 93.2 & 5.5 & 82.2 & 12.3 & 56.2 & 43.8 & 73 & 100.0 \\
\hline & Some Time & 10.2 & 89.8 & 9.1 & 84.1 & 6.8 & 65.9 & 34.1 & 88 & 100.0 \\
\hline & Very Less & 12.5 & 87.5 & 6.3 & 75.0 & 18.8 & 75.0 & 25.0 & 16 & 100.0 \\
\hline & Never & 33.3 & 66.7 & & 100.0 & & 33.3 & 66.7 & 3 & 100.0 \\
\hline \multicolumn{2}{|l|}{ Total } & 9.4 & 90.6 & 7.2 & 82.8 & 10.0 & 62.2 & 37.8 & 180 & 100.0 \\
\hline
\end{tabular}

\section{Perception regarding HIV/AIDS has become Major Problem in Nepal}

The perception of students on whether or not HIV/AIDS is a major problem in Nepal was examined. Overall a majority $(85.6 \%)$ of students $(81.1 \%$ male and $90.0 \%$ female $)$ reported that they "agreed" that HIV/AIDS is a major problem in Nepal, however $14.4 \%$ students $(18.9 \%$ male and $10.0 \%$ female) did not agree on it. A higher percentage of female $(90 \%)$ than male $(81 \%)$ agreed that HIV/AIDS has become major program in Nepal. Similarly, a higher percentage of students who were studying in private school (93\%) than students who were studying in public school (78\%) reported it. 
Table 5: Percentage distribution of students according to their perception regarding HIV/AIDS has become major problem in Nepal

\begin{tabular}{|l|c|c|c|c|c|c|c|c|c|}
\hline \multirow{2}{*}{$\begin{array}{l}\text { Perception regarding } \\
\text { HIV/AIDS has become } \\
\text { major problem in Nepal }\end{array}$} & \multicolumn{3}{|c|}{ Private school } & \multicolumn{3}{c|}{ Public school } & \multicolumn{3}{c|}{ All Total } \\
\cline { 2 - 10 } & Male & Female & Total & Male & Female & Total & Male & Female & Total \\
\hline Agree & 93.3 & 93.3 & 93.3 & 68.9 & 86.7 & 77.8 & 81.1 & 90.0 & 85.6 \\
\hline Disagree & 6.7 & 6.7 & 6.7 & 31.1 & 13.3 & 22.2 & 18.9 & 10.0 & 14.4 \\
\hline Total & $\mathbf{1 0 0}$ & $\mathbf{1 0 0}$ & $\mathbf{1 0 0}$ & $\mathbf{1 0 0}$ & $\mathbf{1 0 0}$ & $\mathbf{1 0 0}$ & $\mathbf{1 0 0}$ & $\mathbf{1 0 0}$ & $\mathbf{1 0 0}$ \\
\hline N & $\mathbf{4 5}$ & $\mathbf{4 5}$ & $\mathbf{9 0}$ & $\mathbf{4 5}$ & $\mathbf{4 5}$ & $\mathbf{9 0}$ & $\mathbf{9 0}$ & $\mathbf{9 0}$ & $\mathbf{1 8 0}$ \\
\hline
\end{tabular}

\section{Conclusion}

Sexual transmitted disease is one of the most important areas of health education. The international conference on population and Development, 1994 stressed that everybody must be informed and has access to safe, effective affordable and acceptable methods of health services to attain a satisfying and safe sexual and reproductive life (UN, 1994).

In the developing country where electronic communication media are not accessible for all parts of the country, formal or informal education instructor can spread their information and knowledge about sexual transmitted disease and HIV/AIDS. It is important to access the current level of knowledge and attitude of secondary level school students on sexually transmitted disease and HIV/AIDS. Inconclusion,finding out knowledge and attitude towards sexual transmitted disease and HIV/AIDS made it clear that they need a specific package of education in school curriculum so that they could gain more information about sexual transmitted disease and HIV/AIDS. Furthermore, teachers who are responsible for day-to-day teaching to the subject matter of sexual transmitted disease and HIV/AIDS in the school seems to be likely poor on subject matter or they might have hesitation and shyness during the lesson. This can be indicated based our findings that reveal the students do not have sufficient knowledge towards sexually transmitted disease and HIV/AIDS related matter. So, teachers should encourage students to discuss on the subject matter not only in school environment, but also in family as well as societal circles. 


\section{References}

Alhasawi, A. Grover, S.B. Sadak, A. Ashoor, I. Alkhabbaz, I. Almasri, S. (2019). Assessing HIV/AIDS Knowledge, Awareness and Attitudes among senior High School Students in Kuwait.Medical Principles and Practice, 28, 470-476.

DeCherney, A. H., Nathan, L. Goodwin, T.M.,Laufer, N. (2007).Current diagnosis $\&$ treatment Obstetrics \& Gynecology. 10th edition. USA: The McGaw-Hill Companies.

Jain, M.A., Jain, S.M.,Patil, S. \& Bang, A. (2016).A study on knowledge, attitude and practice of sexually transmitted diseases and HIV in adolescent population in Wardha, Maharashtra, India.International Journal of Contemporary Pediatrics, 3, 367-72.

Murray, P. R, Rosenthal, K. S.Pfaller, M. A. (2013). Medical Microbiology. 7th ed. St Louis, Mo: Mosby

Poudel et.al. (2015). Knowledge, attitude and practice about STD/HIV AIDSamong the health attendant staffs of a Tertiary Level hospital of Eastern Nepal.Health Renaissance 13(3), 95-106.

United Nations, (1994).Adolescents Reproductive Behaviour, Evidence from Development Countries Population Studies. New York. 